Provided for non-commercial research and education use. Not for reproduction, distribution or commercial use.

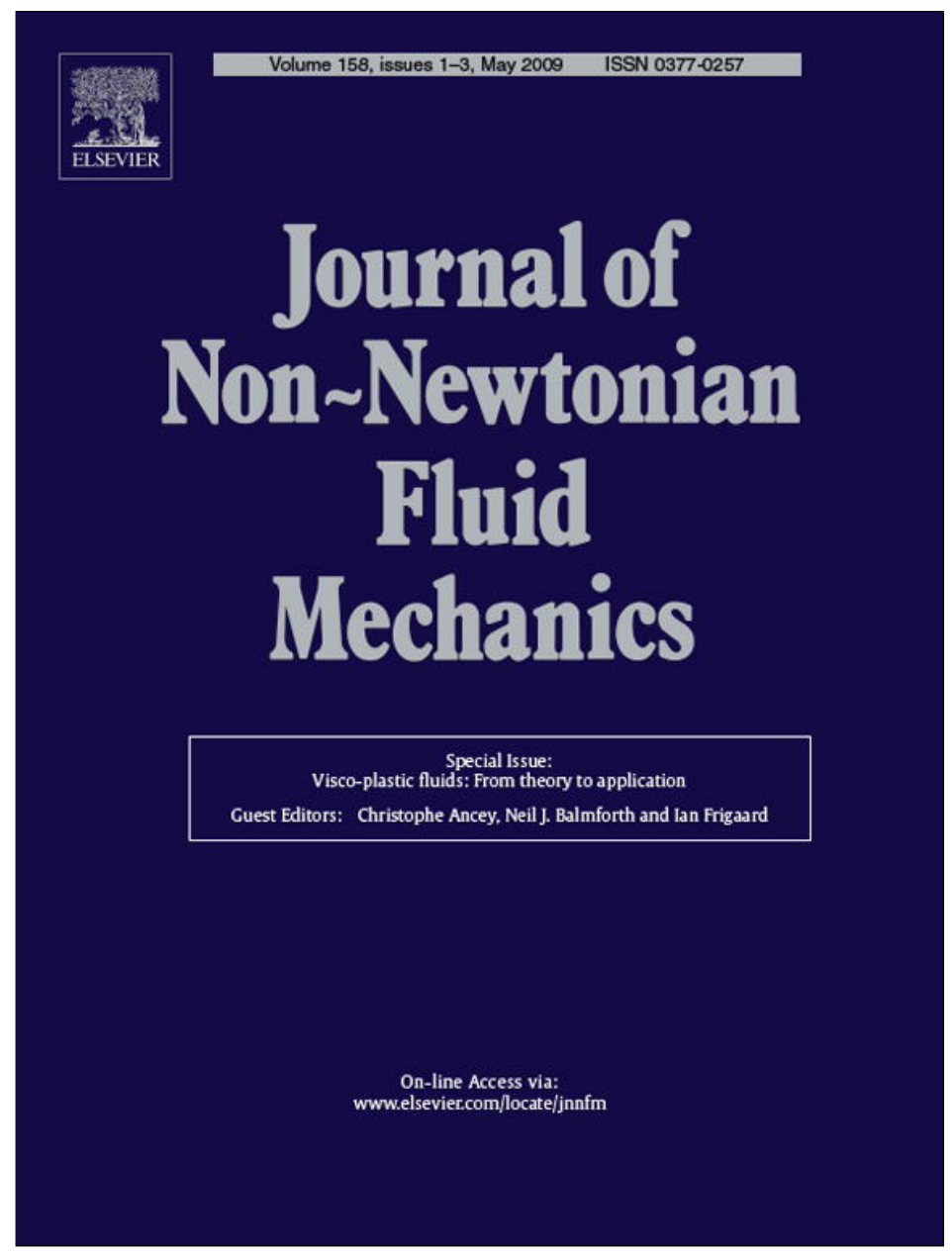

This article appeared in a journal published by Elsevier. The attached copy is furnished to the author for internal non-commercial research and education use, including for instruction at the authors institution and sharing with colleagues.

Other uses, including reproduction and distribution, or selling or licensing copies, or posting to personal, institutional or third party websites are prohibited.

In most cases authors are permitted to post their version of the article (e.g. in Word or Tex form) to their personal website or institutional repository. Authors requiring further information regarding Elsevier's archiving and manuscript policies are encouraged to visit:

http://www.elsevier.com/copyright 


\title{
Slumps of viscoplastic fluids on slopes
}

\author{
A.J. Hogg*, G.P. Matson \\ Centre for Environmental \& Geophysical Flows, School of Mathematics, University of Bristol, University Walk, Bristol BS8 1TW, UK
}

\section{A R T I C L E I N F O}

\section{Article history:}

Received 1 May 2008

Received in revised form 23 June 2008

Accepted 7 July 2008

\section{Keywords:}

Viscoplastic fluid

Yield stress

Herschel-Bulkley

Dam-break

Free-surface flow

Shallow flow

Lambert- $W$ function

\begin{abstract}
A B S T R A C T
Free-surface slumps of viscoplastic fluid, modelled using a Herschel-Bulkley constitutive law, are studied as they flow from rest, behind a rapidly removed dam, along an inclined two-dimensional channel. These dam-break flows are eventually arrested and attain a final, static state in which the streamwise pressure gradient and the along-slope component of gravitational acceleration are balanced by the yield stress. The shapes of the arrested free surfaces are compactly represented as Lambert- $W$ functions and are characterised by two dimensionless parameters that measure the magnitude of the slope relative to the initial aspect ratio of the release and the magnitude of the yield stress relative to the weight of fluid layer. These states are only attained asymptotically for long times after the release and perturbations to the final profiles are shown to decay as $1 / t^{n}$, where $n$ is the power index in the Herschel-Bulkley model. This analysis requires careful formulation, because, formally, within a diminishing boundary layer close to the front of the motion, the size of the perturbation exceeds the arrested state. Thus, while straightforward linearisation of the governing equation is possible within the bulk of the flow outside of the boundary layer, this must be matched asymptotically to the solutions within the boundary layer close to the front. The presence of this region does not obviate computation of the rate of approach to the arrested state, but is required to permit complete calculation of the evolving shape of the free surface.
\end{abstract}

(c) 2008 Elsevier B.V. All rights reserved.

\section{Introduction}

Many materials such as muds, volcanic lava, concrete and various food stuffs exhibit mechanical features typical of viscoplastic fluids (see [1] for a recent comprehensive review). These materials only flow once a yield stress has been exceeded and exhibit a non-linear dependence of shear stress upon the rate of strain. It is common to adopt an empirical constitutive law to model the flow of such materials: for example, the Herschel-Bulkley model, employed in the analysis below, captures the key features of viscoplastic flow in terms of three empirically fitted parameters. However it is important to bear in mind that this, and other similar constitutive laws, are not explicitly linked to any microscopic model of the material. For instance, concentrated particulate suspensions show evidence of yielding as the configuration of the particles changes upon the action of shear and the inter-particle contacts are rearranged, but currently there is not a widely accepted mathematical model to link this micro-mechanics to the bulk motion. The concept of a yield stress, therefore, is often interpreted as an extrapolation of

\footnotetext{
* Corresponding author. Tel.: +44 1179287987; fax: +44 1179287999. E-mail address: a.j.hogg@bris.ac.uk (A.J. Hogg).
}

measured shear stresses to the case of vanishing rates of strain [1].

Free surface flows of viscoplastic materials have wide application to geophysical and industrial situations [1-3] and in recent years considerable progress has been made in developing consistent mathematical models of their motion when the flows are relatively shallow and predominantly parallel to the underlying boundary [4]. Viscoplasticity affects these flows in important ways: first the shear stresses developed within the flow diminish as the free surface is approached and so fluid close to the surface must exhibit 'unyielded' behaviour. Furthermore it is possible that the yield stress is nowhere exceeded within the material, in which case the motion is arrested. This means that flows evolve temporally and spatially from a source, finally arresting to form a static layer.

The Bostwick consistometer features flows with this kind of behaviour: it is a device used to investigate the flow and arrest of food stuffs [5] and to deduce their 'viscosity' and yield stress. The material to be measured is loaded behind a dam in a twodimensional channel and the flow is initiated by its rapid removal. Measurements are taken of the distance that the material has propagated along the channel after a fixed period of time (typically $30 \mathrm{~s}$ ) and then the rheological properties are deduced. The Bostwick consistometer, and its axisymmetric counterpart, the Adams consistometer [6], as well as the generic slump test for concrete, are therefore simple devices for exploring the rheology of viscoplastic 
materials-but the relationship between the measurements they produce and the underlying rheology is non-trivial [5,7]. Part of the difficulty is that the rheology is attempted to be deduced from a single measurement of the length slumped after a certain time (the 'Bostwick length'), whereas Herschel-Bulkley models of viscoplastic materials feature three empirical parameters. Balmforth et al. [7] explored the possibility of deducing extra information by taking measurements at more than one time: an alternative strategy might be to repeat the test with different channel inclinations.

Flows generated by the instantaneous release of an initially stationary material behind a dam ('dam-break' flows) are an important fundamental problem in fluid modelling, not just because of their direct application, but also because they are temporally and spatially evolving and provide considerable insight into the balance of forces driving the motion. They have been a focus of much recent research and yield results that can be compared directly to experiments and to numerical simulations of the motion. In fact the various dam-break solutions provide important test cases for numerical solvers, which are then applied to more complicated problems. Important studies of this type of motion have included: inertially dominated flows [8-10], viscously dominated flows [11,12], the collapse of granular columns [13-15] and the flow of viscoplastic fluids $[7,16,17]$.

The transient motion of relatively thin and slow moving slumps of viscoplastic material down slopes have been analysed theoretically by a number of researchers to reveal the rate of propagation and the form of the final arrested state. Liu and Mei [18] studied the transient slump into a channel that has a pre-existing layer of fluid and they showed how the slump is progressively slowed and then finally arrested as the driving forces are no longer able to surmount the yield stress. Huang and Garcia [16] analysed the motion along an initially fluid-free channel: their calculations revealed that strong curvatures in the free surface are only found close to the propagating front and this permitted a simplified analysis of the motion in which the front was handled separately and matched to the interior. Their analysis is appropriate for relatively steep slopes and these results are recovered in this study within an appropriate asymptotic regime that is identified below. A similar mathematical model was presented by Balmforth et al. [19], who also treated three dimensional flows down planes and over gently varying topography to calculate the lateral and downstream spreading.

Flows of yield stress materials down slopes have also been investigated experimentally. Often these relatively small-scale experiments have employed clay dispersions and have measured the flow speed and runout length, following the release of a volume of material (see, for example [16,18-21]). Liu and Mei [18] released mud into a sloping channel and showed that the profile of the material, once the flow had arrested, was quite similar to the theoretical predictions, for which the rheology of the mud was independently determined. Huang and Garcia [16] studied the transient problem by measuring the position of the front of the flow at various times after release and found that its position was reasonably well predicted by their theory. Relatively little attention has been paid to the approach of these flows to their final, static states, although slumps of concentrated suspensions of particules, possibly released with significant inertia, have been shown to arrest abruptly [22,23]. A recent study, however, has studied the flow of Carbopol down an inclined plane and taken measurements of the position of the front over relatively long timescales $\left(>10^{5} \mathrm{~s}\right.$ ) [21]. Most notably for the current contribution, Cochard [21] appears to show that the final state is approached asymptotically.

In this study we analyse the flow along an inclined plane of a viscoplastic material, released from rest behind a instantaneously removed lockgate. The fluid is assumed to form a relatively thin film so that the predominant forces driving the motion along the plane are the streamwise pressure gradients due to variations in the thickness of the film, supplemented, or moderated, by gravitational acceleration depending on whether the plane is inclined downwards or upwards and resisted by 'viscous-like' stresses. We neglect the inertia of the flowing material. Our particular focus is to analyse the approach of the mobile layers towards the final arrested state in which the pressure gradient, gravitational acceleration and yield stress are in balance. Our key result is that this final state is only approached asymptotically in time, with perturbations decaying algebraically and at a rate that we calculate analytically. This result has immediate implications for the interpretation of measurements from devices such as the Bostwick consistometer, but also explains what has been observed in numerical computations $[16,19]$. It is the counterpart of what has been established on horizontal surfaces [17]. The methodology used to establish the results in this paper is rather more careful than what has been used in previous studies. As the final state is approached, we show that the precise balance in the dynamics close to the front of the motion is different from those in the interior. This is handled analytically by introducing matched asymptotic expansions between the interior and the region close to the front. It is noteworthy that although from different contexts and thus having very different structures, this approach of matching an interior solution to a model with a different balance of forces close to the front of the flow where the depth of the moving fluid becomes small, has been employed before. For example, Hogg and Pritchard [10] examined the effects of drag on an inertial dam-break flow, Hocking [24] showed the effects of surface tension on a viscous flow and, as described above, Huang and Garcia [16] demonstrated that for sufficiently steep slopes the 'viscous' stresses due to the curvature of the interface may be neglected, apart from within regions close to the front. In this study we show that this new region is sub-dominant and thus while it controls the profile of the interface close to the front, it does not contribute to the leading order expression for its speed, unless the yield stress is vanishingly small and the fluid is flowing up an inclined plane to approach a state in which its final interface is horizontal.

The paper is structured as follows. First we formulate the problem and identify the dynamical regime under consideration (Section 2). Two important dimensionless parameters emerge: these measure the inclination of the plane and the magnitude of the yield stress. The final arrested state in then constructed in Section 3 . This has been formulated before (see, for example $[2,18,19]$ ). Here we write it in compact form, expressing the profile in terms of a Lambert- $W$ function (see Appendix A). There are two real-valued branches of this function and we show that these correspond to the static profile on upwardly and downwardly sloping planes. This compact form significantly aids the analysis that follows in Section 4 , in which we calculate the approach to the arrested state. We study the perturbations to the final state and examine how they vary with time. This analysis requires a careful consideration of the magnitude of the final state and the perturbation close to the front and the development of asymptotic expansions that match between the frontal region and the interior. Throughout Sections 3 and 4 we treat two-dimensional slumps along planes inclined upwards and downwards. A different analytical approach is required for some of the analysis of the motion along upwardly inclined planes. This arises because the flows are resisted by gravity in addition to the viscous stresses and even without a yield stress, the material can form a trivial arrested state with a horizontal surface. Also throughout this paper, we develop asymptotic expressions for various quantities that emerge from the analysis to show how they depend on slope and yield stress. Finally we summarise the results and draw some conclusions in Section 5. 


\section{Formulation}

We analyse the two-dimensional, gravitationally driven, freesurface slump and arrest of a volume of fluid with Herschel-Bulkley rheology on an inclined plane to calculate how far the fluid propagates before it arrests and the rate at which the arrested state is approached. The fluid is released from rest within a reservoir, the lockgate of which is instantaneously removed at $t=0$ to generate the free-surface flow along the inclined plane. As it propagates, the thickness of the flowing layer thins and eventually the driving forces, which are the streamwise gradients of the hydrostatic pressure and gravitational acceleration, if the plane is sloping downwards, are unable to surmount the yield stress of the material and thus the motion is arrested. However as we will demonstrate below the arrested state is only approached asymptotically, as $t \rightarrow \infty$.

The fluids under consideration in this study are characterised by a Herschel-Bulkley rheology and thus the shear stress tensor, $\tau_{i j}$, is related to the strain rate tensor, $\dot{\gamma}_{i j}$, by

$\tau_{i j}= \begin{cases}\left(\tau_{0}+\mu_{n}\left|\dot{\gamma}_{i j}\right|^{n}\right) \dot{\gamma}_{i j} /\left|\dot{\gamma}_{i j}\right|, & \left|\tau_{i j}\right|>\tau_{0}, \\ 0, & \left|\tau_{i j}\right|<\tau_{0},\end{cases}$

where $\tau_{0}$ is the yield stress, $\mu_{n}$ is the consistency and $n$ is the index of the Herschel-Bulkley material. (In this expression $|\cdot|$ denotes the second invariant of the tensor.) The flow propagates along a plane inclined at an angle $\theta$ to the horizontal and we orientate the coordinate axes so that the $x$-axis is the streamwise coordinate along the plane and the $z$-axis is perpendicular to the plane (see Fig. 1). The fluid is of density $\rho$ and gravitational acceleration is denoted by $g$. The thickness of the fluid is denoted by $h(x, t)$ and the initial distribution of fluid within the reservoir is given by

$h(x, 0)= \begin{cases}h_{0}+x \tan \theta, & 0<x<x_{0}, \\ 0, & x_{0}<x .\end{cases}$

The motion is characterised by five dimensionless parameters: the aspect ratio $\epsilon=h_{0} / x_{0}$; the Reynolds number, $R e=\rho U x_{0} / \mu$, where $\mu$ is the effective viscosity for a Herschel-Bulkley fluid, $\mu=\mu_{n}\left(U / h_{0}\right)^{n-1}$ and $U=\rho g \cos \theta h_{0}^{3} /\left[\mu x_{0}\right]$ is a velocity scale for the motion; the rheological index of the Herschel-Bulkley fluid, $n$; and of most significance for the analysis that follows, the slope, $S$ and the Bingham number, $B$, defined by

$S=\frac{x_{0} \tan \theta}{h_{0}}$ and $B=\frac{\tau_{0}}{\epsilon \rho g h_{0} \cos \theta}$.

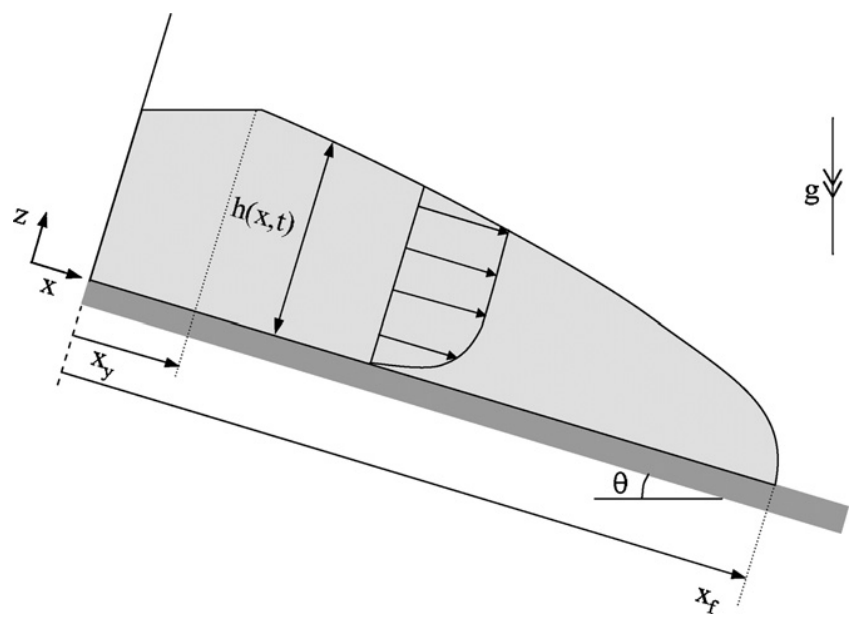

Fig. 1. The configuration of the flow.
Note that $S>-1$ by virtue of the initial condition and that the parameter, $B$, measures the magnitude of the yield stress relative to the weight of the flowing layer. The dynamical regime under consideration corresponds to relatively thin flows $(\epsilon \ll 1)$, so that the pressure is hydrostatic to leading order, and to situations with negligible inertia $\left(\epsilon^{2} R e \ll 1\right)$ so the motion is dynamically controlled by a balance of downslope acceleration and streamwise pressure gradients with the divergence of viscous stresses. (This balance yields the velocity scale, $U$, introduced above.) In this regime the shear stress is then given to leading order by

$\tau_{x z}=\rho g\left(\sin \theta-\cos \theta \frac{\partial h}{\partial x}\right)(h-z)$,

and motion occurs provided the shear stress exceeds the yield stress at some point within the flow, a condition that requires

$\rho g \cos \theta h\left(\tan \theta-\frac{\partial h}{\partial x}\right)>\tau_{0}$.

Provided the yield stress is exceeded, then to leading order the down-slope velocity exhibits a shearing region close to the basal boundary, overridden by a plug-like region (see, for example [18]). At the yield surface between the two, the velocity field and shear stress are continuous. As demonstrated by Balmforth and Craster [4], this interface is a 'fake' yield surface above which there is 'pseudo-plug flow', with the deviation from a truly rigid, plug-like region being represented at higher asymptotic orders.

We introduce the following dimensionless variables

$\left\{x, z, h, t, \tau_{x y}\right\}=\left\{x_{0} x^{\prime}, h_{0} z^{\prime}, h_{0} h^{\prime}, \frac{x_{0} t^{\prime}}{U}, \rho g \cos \theta h_{0} \tau_{x y}^{\prime}\right\}$.

Henceforth all variables will be assumed to be dimensionless and for notational clarity, the primes will be dropped.

The equation governing the evolution of the height, $h(x, t)$, is derived by integrating the downslope velocity field over the depth of the fluid layer to calculate the volume flux per unit width and then the temporal rate of change of $h(x, t)$ is equal to the spatial divergence of the volume flux. Following [18], this gives

$$
\begin{aligned}
\frac{\partial h}{\partial t}= & -\frac{n}{1+2 n} \frac{\partial}{\partial x}\left[\left(S-\frac{\partial h}{\partial x}\right)^{-2}\left(h\left(S-\frac{\partial h}{\partial x}\right)-B\right)^{1+1 / n}\right. \\
& \left.\times\left(h\left(S-\frac{\partial h}{\partial x}\right)+\frac{n}{1+n} B\right)\right] .
\end{aligned}
$$

This equation is to be solved in the region where the yield stress is exceeded, given in dimensionless form by

$S-\frac{\partial h}{\partial x}>\frac{B}{h}$.

The yielded region ranges between the front, $x_{\mathrm{f}}(t)$, where $h\left(x_{\mathrm{f}}, t\right)=0$ and either a yield point, $x_{\mathrm{y}}(t)$, where $h\left(x_{\mathrm{y}}, t\right)=h\left(x_{\mathrm{y}}, 0\right)$, or the back of the lockgate $(x=0)$, if the entire fluid is in motion. In both of these cases we also demand that at $x=x_{\mathrm{y}}(t)$ or $x=0$, respectively, there is no flux of fluid per unit width, given by

$S-\frac{\partial h}{\partial x}=\frac{B}{h}$.

The flow arrests when all of the material exhibits the balance between gravitational acceleration, pressure gradient and yield stress, given by (2.9).

In formulating the asymptotic expansions that lead to this mathematical model of the motion, we have implicitly assumed that $S=O(1)$, which implies that the gradient of the slope is comparable to the initial aspect ratio and this is assumed to be small. Thus to leading order, the dimensionless shear stress is given by 
$\tau_{x z}=(S-\partial h / \partial x)(h-z)$. We note that flows over horizontal planes may still be modelled in this formulation by taking the limit $S \rightarrow 0$ and thus the dimensionless shear stress is given by $\tau_{x z}=$ $-\partial h / \partial x(h-z)$ [17]. Flows down steep slopes $(S \gg 1)$ may also be studied: while $S \gg|\partial h / \partial x|$ throughout most of the current, it is not the case close to the front of the current, where it will be shown below that $\partial h / \partial x$ becomes unbounded and thus we retain both terms in the expression for the dimensionless shear stress. Some previous studies of flows down steep slopes have employed the approximation that $\tau_{x z}=S(h-z)$ (e.g. [16]) and this means that the front is no longer identified as the the position where the height of the flowing layer vanishes, but rather is found by enforcing conservation of mass throughout the flow. Such models do not resolve the shape of the interface close to the front, but remain accurate because the frontal region is of negligible extent. Finally we note that (2.7) and (2.9) predict the divergence of the gradient of the interface as the front is approached (see below). This is a common feature in models of thin film flows of viscous fluids. The divergence may be regularised by reinstating dynamical terms that are no longer asymptotically small, or by including physical processes that do not feature in the original model, such as surface tension. The analysis of viscous flows in the absence of a yield stress indicates that the inclusion of capillarity does not significantly modify the predictions of the motion; rather it introduces a new subdominant asymptotic region close to the front [24]. We emphasise that capillary effects will be neglected in this paper.

The evolution of the flow from initial conditions (2.2) may be calculated by numerically integrating the partial differential Eq. (2.7). To this end it is useful to introduce a rescaled coordinate

$\xi=\frac{\left[x-x_{\mathrm{y}}(t)\right]}{\left[x_{\mathrm{f}}(t)-x_{\mathrm{y}}(t)\right]}$,

so that the computational domain is $0 \leq \xi \leq 1$. Considerable care is needed in adequately resolving the height close to the front and to the yield point, where the curvature is high and the local power series expansions of $h(\xi, t)$ exhibit fractional powers [17]. In this study we adopt the numerical scheme proposed by [7]: the rescaled spatial coordinate is discretised onto a non-uniform grid with grid-points clustered at the front of the flow $(\xi=1)$, typically according to $\xi_{i}=1-[(N-i) /(N-1)]^{3}$ with $i=1 \leq i \leq N$. The speeds of the front and the yield point are determined by evaluating the governing equation at $\xi=0$ and 1 , respectively. Time is stepped forward using a fifth-order backward differentiation formula (Gear's method). The results shown in Section 4 with $N=200$ are typically produced in runtimes of a few minutes.

In this contribution we first analyse the arrested state and then show how to calculate analytically the approach to that arrested state.

\section{The arrested state from dam-break initial conditions}

\subsection{Flows down inclined planes $(S>0)$}

From dam-break initial conditions (2.2), the fluid attains an arrested profile $h_{\infty}(x)$, which satisfies

$h_{\infty}\left(S-\frac{\partial h_{\infty}}{\partial x}\right)=B$.

This may be integrated subject to the condition at the front, $x_{f_{\infty}}$, namely $h_{\infty}\left(x_{\mathrm{f} \infty}\right)=0$ to give

$h_{\infty}(x)=\frac{B}{S}\left[1+W_{0}\left(-\mathrm{e}^{-1+S^{2}\left(x-x_{\mathrm{f} \infty}\right) / B}\right)\right]$,

where $W_{0}$ denotes the Lambert- $W$ function (see Appendix A and [25]). This expression produces identical arrested profiles to those presented by [2,18], but here we have expressed $h_{\infty}(x)$ as an explicit function and this compact form is useful in the analysis that follows. The expression is valid for $x_{\mathrm{y} \infty} \leq x \leq x_{\mathrm{f} \infty}$, where $x_{\mathrm{y} \infty}$ denotes the arrested position of the yield point. It is possible that the entire fluid was in motion before arrest, in which case the active region is $0 \leq x \leq x_{\mathrm{f} \infty}$-and this case will be treated below.

We may evaluate $x_{\mathrm{y} \infty}$ and $x_{\mathrm{f} \infty}$ by requiring that $h_{\infty}\left(x_{\mathrm{y} \infty}\right)=$ $h\left(x_{\mathrm{y} \infty}, 0\right)$, which is represented by

$1+S x_{\mathrm{y} \infty}=\frac{B}{S}\left[1+W_{0}\left(-\mathrm{e}^{\left.-1+S^{2}\left(x_{\left.y_{\infty}-x_{\mathrm{f}_{\infty}}\right) / B}\right)\right],}\right.\right.$

and by enforcing conservation of volume per unit width, which gives

$\int_{x_{\mathrm{y} \infty}}^{x_{\mathrm{f} \infty}} h_{\infty} \mathrm{d} x=\left(1-x_{\mathrm{y} \infty}\right)+\frac{S}{2}\left(1-x_{\mathrm{y} \infty}^{2}\right)$.

Thus by employing (A.2) to evaluate (3.4) and using (3.3), we deduce that the active region $x_{\mathrm{a} \infty} \equiv x_{\mathrm{f} \infty}-x_{\mathrm{y} \infty}$ is given by

$x_{\mathrm{a} \infty}=\frac{(S+1)^{2}}{2 B}$,

and thence the arrested yield point is given by

$x_{\mathrm{y} \infty}=\frac{1}{S}\left[\frac{B}{S}\left(1+W_{0}\left(-\mathrm{e}^{-1-S^{2}(S+1)^{2} /\left[2 B^{2}\right]}\right)\right)-1\right]$.

Whether the entire material takes part in the slump is determined by the magnitudes of the Bingham number, $B$ and the slope, $S$. In particular there is a critical Bingham number, $B_{\mathrm{c}}(S)$, for which $x_{\mathrm{y} \infty}=0$, such for that $B>B_{\mathrm{c}}$ there exists an arrested interior yield point. This critical Bingham number is implicitly determined by

$0=\frac{B_{\mathrm{C}}}{S}\left[1+W\left(-\mathrm{e}^{-1-S^{2}(S+1)^{2} /\left(2 B_{\mathrm{C}}^{2}\right)}\right)\right]-1$

and plotted in Fig. 2 . In the regime $S \ll 1$, we determine that

$B_{\mathrm{c}}=\frac{1}{3}+\frac{7 \mathrm{~S}}{12}+\cdots$,

which is consistent with the critical Bingham number determined for dam-break flows over a horizontal surface [17]. Furthermore for

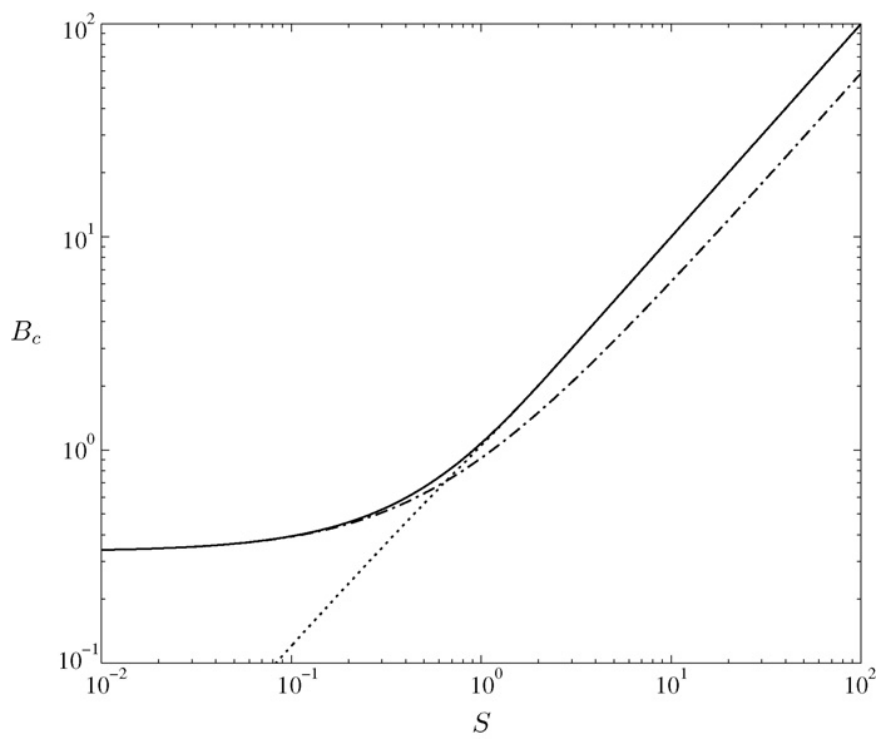

Fig. 2. The critical Bingham number, $B_{\mathrm{c}}$, as a function of the slope, $S$, for downslope slumps. For $B<B_{\mathrm{c}}$ all of the fluid in the reservoir is set in motion during the slump, whereas for $B>B_{c}$ only part of the fluid flows. Also plotted are the asymptotic expressions for the dependence of $B_{\mathrm{c}}$ upon $S$ in the regimes $S \ll 1(\cdots)$ and $S \gg 1$ $(-\cdot-\cdot)$. 
relatively steep slopes, $S \gg 1$, we find that

$B_{\mathrm{C}}=S\left(1+\mathrm{e}^{-1-(S+1)^{2} / 2}+\cdots\right)$

We observe in Fig. 2 that in their relevant regimes these asymptotic expressions accurately capture the numerically computed values.

If $B<B_{\mathrm{c}}$ then at some time during the flow, the yield position has moved back to the rear wall and thereafter all of the fluid is in motion. In this case, the arrested position of the front is determined by using mass conservation and integrating the profile (3.2) to give

$$
\begin{aligned}
& \frac{B}{S} x_{\mathrm{f} \infty}-\frac{B^{2}}{S^{3}}\left[\frac{1}{2}+\frac{1}{2}\left(W_{0}\left(-\mathrm{e}^{-1-S^{2} x_{\mathrm{f} \infty} / B}\right)\right)^{2}+W_{0}\left(-\mathrm{e}^{-1-S^{2} x_{\mathrm{f} \infty} / B}\right)\right] \\
& \quad=1+\frac{S}{2} .
\end{aligned}
$$

It is also possible to deduce asymptotically the arrested front and yield positions in the regime $B>B_{\mathrm{c}}$. This gives two possibilities: when $S(1+S) \gg B>B_{\mathrm{c}}$ then

$x_{\mathrm{y} \infty}=\frac{B}{S^{2}}+\cdots \quad$ and $\quad x_{\mathrm{f} \infty}=\frac{(S+1)^{2}}{2 B}+\cdots$

Conversely when $B \gg S(S+1)$ and $B>B_{\mathrm{c}}$, we find that

$x_{\mathrm{y} \infty}=1-\frac{(S+1)^{2}}{3 B}+\cdots$ and $x_{\mathrm{f} \infty}=1+\frac{(S+1)^{2}}{6 B}+\cdots$

Once again (3.12) is consistent with results in a horizontal channel[17]. In the regime $B_{\mathrm{c}}>B \gg S^{2}$

$x_{\mathrm{f} \infty}=\left(\frac{9(1+S / 2)^{2}}{8 B}\right)^{1 / 3}+\cdots$,

while when $B_{\mathrm{c}}>B$ and $S^{2} \gg B$

$x_{\mathrm{f} \infty}=\frac{S}{B}\left(1+\frac{S}{2}\right)$.

We plot the dependence of $x_{\mathrm{f} \infty}$ and $x_{\mathrm{y} \infty}$ upon $B$ and $S$ in Figs. 3 and 4, along with some examples of the arrested profiles (Fig. 5). The latter result, (3.14), recovers the expression derived by Huang and Garcia [16] for flows down relatively steep slopes.

\subsection{Flows up inclined planes $(0>S>-1)$}

When the fluid is released from rest behind the dam into an upwardly sloping flume it will also attain an arrested final state in which the hydrostatic pressure gradients are insufficient to overcome the combination of the yield stress and gravitational acceleration. Upslope slumps are somewhat different from their downslope counterparts because they can still be arrested in the absence of a yield stress $(B=0)$, in which case the free surface of the final state is horizontal. In this situation the final profile is given by

$h_{\infty}=\sqrt{-2 S-S^{2}}+S x$,

and the length of the intrusion is $x_{\mathrm{f}_{\infty}}=\sqrt{(2+S) /(-S)}$. For materials with a non-vanishing yield stress $(B>0)$, the arrested profile also satisfies (3.1). However in this case its solution may be written

$h_{\infty}(x)=\frac{B}{S}\left[1+W_{-1}\left(-\mathrm{e}^{-1+S^{2}\left(x-x_{\mathrm{f} \infty}\right) / B}\right)\right]$,

where $W_{-1}$ denotes the second real-valued, principal branch of the Lambert- $W$ function, defined for $z<-\mathrm{e}^{-1}$ (see Appendix A and [25]). Many of the results of the previous subsection carry over to the regime $-1<S<0$ : inparticular, if $B>B_{c}$ then there exists an
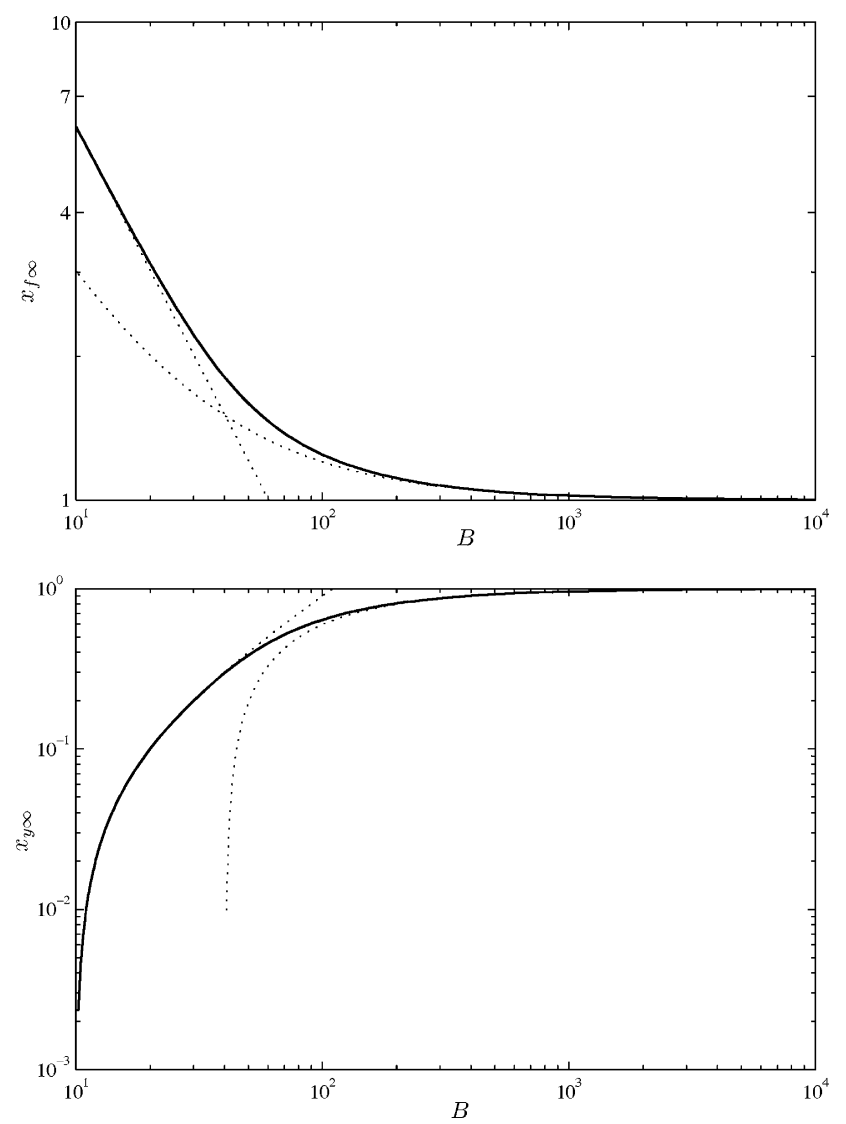

Fig. 3. The position of the arrested front, $x_{\mathrm{f}_{\infty}}$ and yield position, $x_{\mathrm{y}_{\infty}}$, as a function of the Bingham number, $B$ when $S=10$, for which $B_{\mathrm{c}}=10.0000$. Also plotted are the asymptotic approximations for $x_{\mathrm{f} \infty}$ and $x_{\mathrm{y} \infty}$ in the regimes $S(1+S) \gg B \gg B_{\mathrm{c}}$ and $B \gg S(S+1)(\cdots)$.

interior yield point in the arrested state and $x_{\mathrm{a} \infty}$ is given by (3.5) and

$x_{\mathrm{y} \infty}=\frac{1}{S}\left[\frac{B}{S}\left(1+W_{-1}\left(-\mathrm{e}^{-1-S^{2}(S+1)^{2} /\left[2 B^{2}\right]}\right)\right)-1\right]$.

Thus the critical Bingham number for the existence of this yield point is given by (3.7), with the Lambert- $W$ function, $W_{0}$, replaced by the other real-valued branch, $W_{-1}$. We plot $B_{\mathrm{c}}$ as a function of $S$

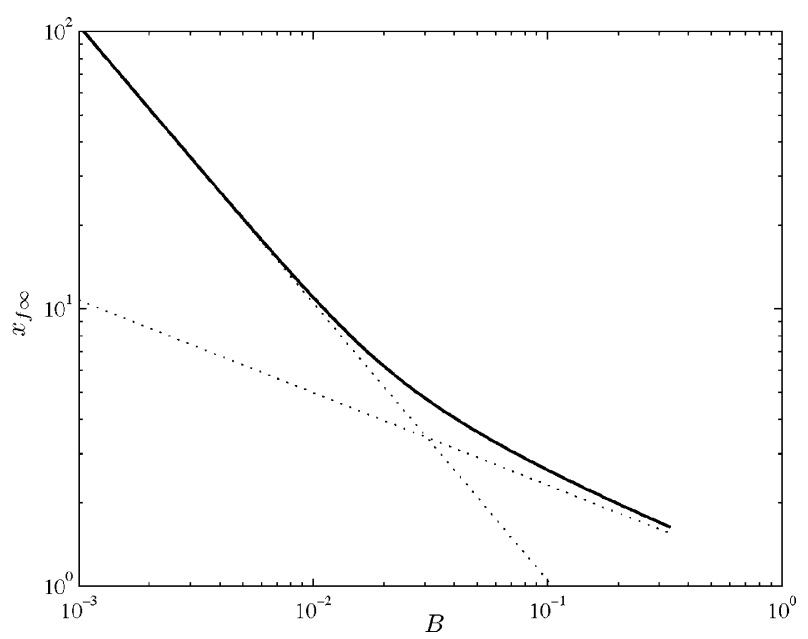

Fig. 4. The position of the arrested front, $x_{\mathrm{f} \infty}$ as a function of the Bingham number, $B$ when $S=0.1$, for which $B_{\mathrm{c}}=0.3936$. Also plotted are the asymptotic approximations for $x_{\mathrm{f} \infty}$ in the regimes $B_{\mathrm{c}} \gg B \gg S^{2}$ and $S^{2} \gg B$ and $B_{\mathrm{c}} \gg B(\cdots)$. 

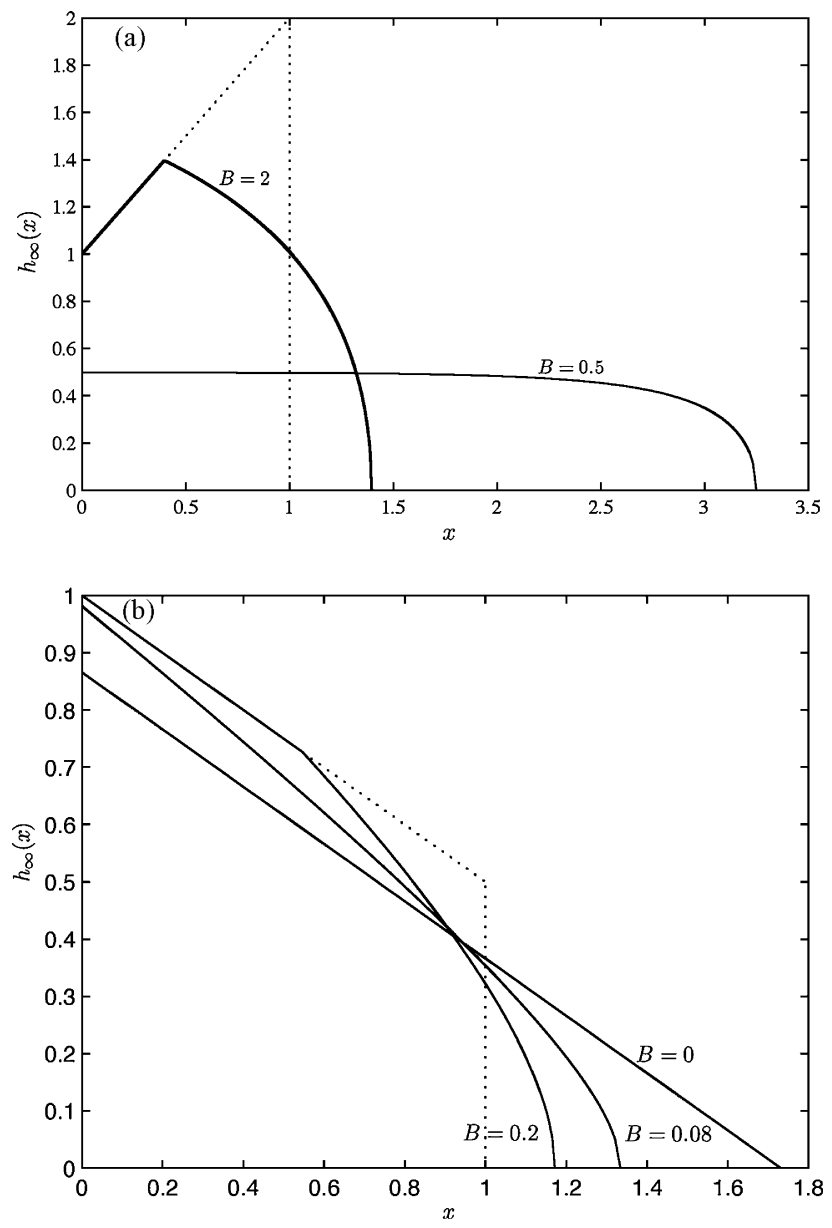

Fig. 5. The arrested profile of fluid $h_{\infty}$ (x) for (a) $S=1$ and $B=0.5,2$; and for (b) $S=-0.5$ and $B=0,0.08,0.2$. Note that when $S=1$, the critical Bingham number $B_{c}=1.0752$ and thus in (a) all of the fluid in the reservoir flows for $B=0.5$, whereas for $B=2$, there is an interior yield position, upstream of which the fluid surface remains horizontal. Further note that when $S=-0.5$, the critical Bingham number $B_{c}=0.09634$ and so in (b), there is only an interior yield point for $B=0.2$. In both (a) and (b), the initial condition is also plotted $(\cdots)$, noting that the axes are aligned so that initially the free surface within the reservoir is horizontal.

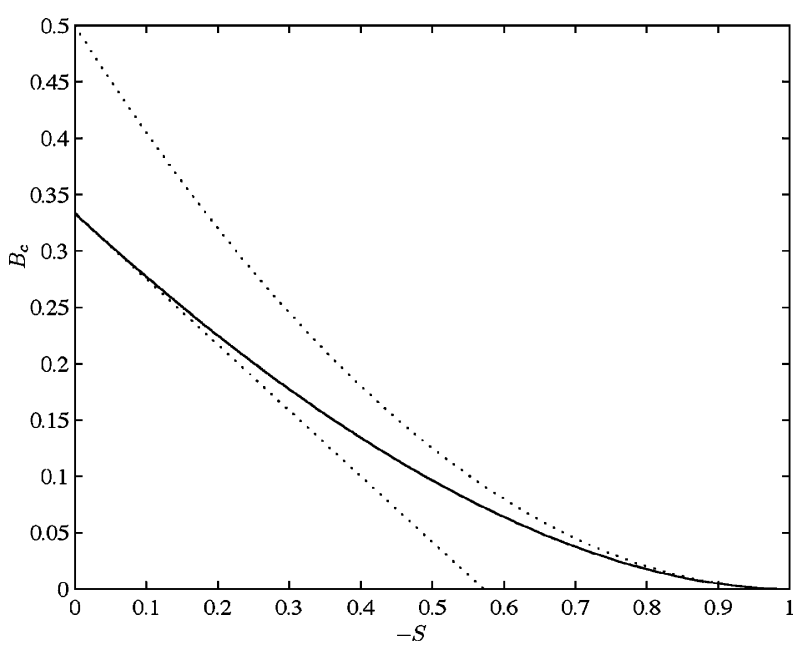

Fig. 6. The critical Bingham number, $B_{\mathrm{c}}$, as a function of the slope, $S$ for upslope slumps. For $B<B_{\mathrm{c}}$ all of the fluid in the reservoir is set in motion during the slump, whereas for $B>B_{\mathrm{c}}$ only part of the fluid flows. Also plotted are the asymptotic expressions for the dependence of $B_{\mathrm{c}}$ upon $S$ in the regimes $|S| \ll 1$ and $0<1+S \ll 1$ $(\cdots)$. in Fig. 6, noting that for $|S| \ll 1$, the asymptotic behaviour is given by (3.8), while in the regime $0<1+S \ll 1$,

$B_{\mathrm{c}}=\frac{1}{2}(1+S)^{2}+\cdots$

When $B<B_{\mathrm{c}}$, all of the fluid is set in motion at some instant before it arrests. In this case the position of the arrested front is given by (3.10), again with the Lambert- $W$ function $W_{0}$ replaced by $W_{-1}$. It is noteworthy that by evaluating (3.10) in the limit $B \rightarrow 0$, we find $x_{\mathrm{f} \infty}=\sqrt{(2+S) /(-S)}$, recovering the result presented above for the slump of a viscous fluid in the absence of a yield stress. There is, however, an important feature that differs between the arrested profiles with and without a yield stress $(B>0$ and $B=0$, respectively). Close to the front $\left(x_{\mathrm{f}_{\infty}}-x\right) / x_{\infty} \ll 1$,

$h_{\infty}=\sqrt{2 B\left(x_{\mathrm{f}_{\infty}}-x\right)}+\cdots$ when $B>0$,

whereas

$h_{\infty}=S\left(x-x_{\mathrm{f} \infty}\right)$ when $B=0$.

This different curvature close to the front will be shown to have a significant effect on the analysis that follows.

\section{Approach to the arrested state}

We numerically integrate the governing equation from initial conditions (2.2) for a Bingham fluid $(n=1)$, slope $S=1$ and (a)
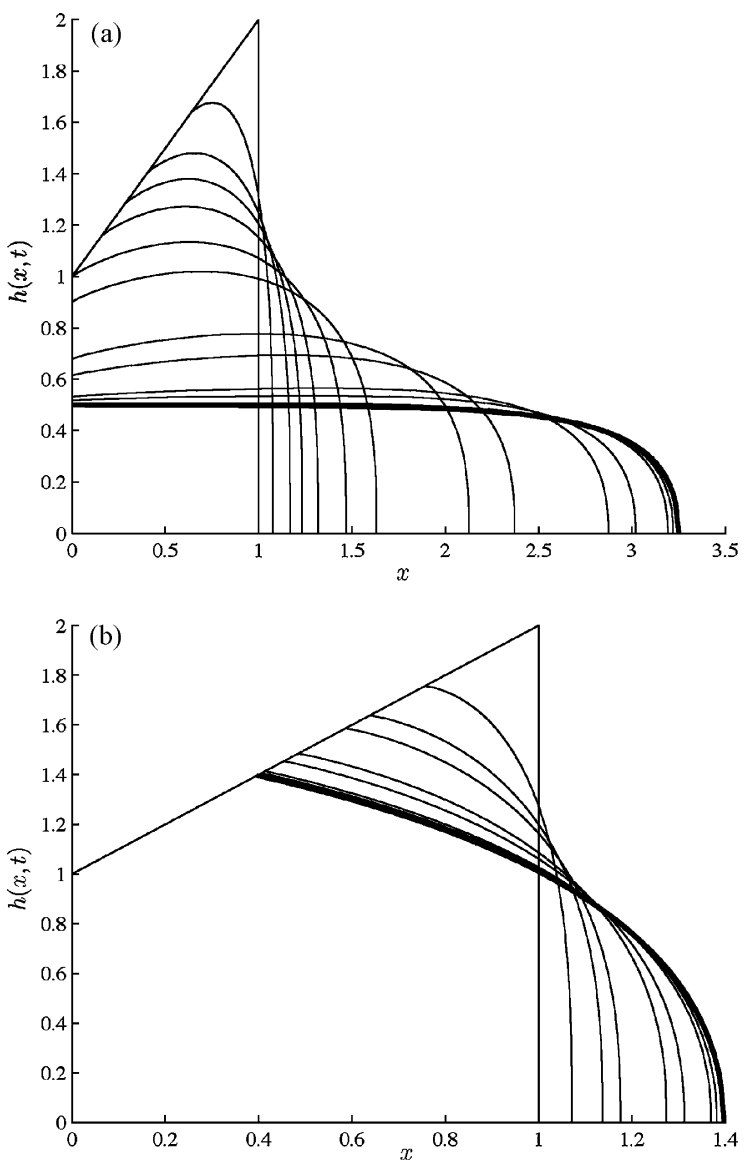

Fig. 7. The evolution of the profile of fluid, $h(x, t)$, released from rest behind a lockgate, and flowing down a slope $(S=1)$ for $(\mathrm{a}) B=0.5$ at times $t=0.01,0.05,0.1,0.2$. $0.4816,1,5,10,50,100,500,1000,5000,10,000$ and (b) $B=2$ at times $t=0,0.01$ $0.05,0.1,0.5,1,5,10,50,100$. The flows approach an arrested state, here plotted with a thicker line. Note that for (a), the yield point reaches the back of the lock $(x=0)$ at $t=0.4816$. 
$B=0.5$ and (b) $B=2$ (see Fig. 7(a) and (b)). When $S=1$, the critical Bingham number for the existence of an interior yield point is $B \equiv B_{\mathrm{c}}=1.0752$. Thus in Fig. 7(a) and $B=0.5$, we observe that the yield point moves backwards to the rear wall, reaching it at $t=0.4816$; thereafter the entire fluid is set in motion. From Fig. 7(a) and (b) it may be observed that the fluid evolves from the initial condition, at rest within the reservoir, and approaches the final arrested state-and it is this evolution that is considered in this section.

We examine how the height of the flowing layer, treated as a function of the rescaled spatial variable, $\xi$, and the positions of the front and yield points approach their arrested states $h_{\infty}, x_{\mathrm{f} \infty}$ and $x_{\mathrm{y} \infty}$, respectively. If $B<B_{\mathrm{c}}$, it is sufficient to set $x_{\mathrm{y}}=0$ for sufficiently long times, however if $B>B_{\mathrm{c}}$ then $x_{\mathrm{y}}(t)$ must always exceed $x_{\mathrm{y} \infty}$. Thus we define the following perturbation variables, $\tilde{h}(\xi, t)$, $\tilde{x}_{\mathrm{f}}(t)$ and $\tilde{x}_{\mathrm{y}}(t)$, to these arrested states

$h(\xi, t)=h_{\infty}(\xi)+\epsilon \tilde{h}(\xi, t), \quad x_{\mathrm{f}}(t)=x_{\mathrm{f} \infty}-\epsilon \tilde{x}_{\mathrm{f}}(t) \quad$ and

$x_{\mathrm{y}}(t)=x_{\mathrm{y} \infty}+\epsilon \tilde{x}_{\mathrm{y}}(t)$,

where $\epsilon$ is an ordering parameter. In the analysis that follows, we assume that $\epsilon \ll 1$, so that deviations from the final states are relatively small. These definitions imply that the length of fluid in motion is given by

$x_{\mathrm{a}}(t)=x_{\mathrm{a} \infty}-\epsilon\left(\tilde{x}_{\mathrm{f}}(t)+\tilde{x}_{\mathrm{y}}(t)\right) \equiv x_{\mathrm{a} \infty}-\epsilon \tilde{x}_{\mathrm{a}}(t)$.
We comment that potentially we should not enforce (4.5) on this perturbation function, $\tilde{h}(\xi, t)$, the governing equation for which is derived by linearisation of (2.7) under the assumption that $h_{\infty} \gg$ $\epsilon \tilde{h}$. This regime can not be guaranteed at this stage of the anlaysis. However we will show below that a more appropriate asymptotic analysis close to the front produces a more complete solution that turns out to be consistent with this boundary condition. If $B>B_{\mathrm{c}}$ we have one further boundary condition, that the height must be equal to the height of fluid in the reservoir at $\xi=0$ for all time, and hence

$\tilde{h}(0, t)=S \tilde{x}_{\mathrm{y}}$.

To solve (4.3) subject to these boundary conditions, we construct a solution in separable form, $\tilde{h}(\xi, t)=A(\xi) G(t), \tilde{x}_{\mathrm{f}}(t)=F(t)$ and $\tilde{x}_{\mathrm{y}}(t)=H(t)$, where $F, G, H \rightarrow 0$ as $t \rightarrow \infty$. Upon substitution into (4.3) and (4.4) it is found that $F(t), G(t)$ and $H(t)$ must differ only by a multiplicative constant and thus we write

$\tilde{h}(\xi, t)=S E(\xi) F(t)$

$\tilde{x}_{\mathrm{f}}(t)=F(t)$,

$\tilde{x}_{\mathrm{y}}(t)=\lambda F(t)$

where $\lambda$ is a constant to be found. If $B<B_{\mathrm{c}}$ then $\lambda=0$ because the yield point reaches the back wall within finite time and thereafter $x_{\mathrm{y}}=0$. Substitution of these separable forms (4.7)-(4.9) into (4.3) and separation of terms yields

$\frac{1}{F^{1+1 / n}} \frac{\mathrm{d} F}{\mathrm{~d} t}=-\frac{n}{1+n} \frac{\epsilon^{1 / n} B^{1 / n}}{x_{\mathrm{a} \infty}} \frac{(\mathrm{d} / \mathrm{d} \xi)\left[h_{\infty}^{2}\left(\left(S E / h_{\infty}\right)+\left(1-\left(h_{\infty} S / B\right)\right)\left((1+\lambda) / x_{\mathrm{a} \infty}\right)-\left(S h_{\infty} / B x_{\mathrm{a} \infty}\right)(\mathrm{d} E / \mathrm{d} \xi)\right)^{1+1 / n}\right]}{S E-\left(B / h_{\infty}\right)\left(1-\left(h_{\infty} S / B\right)\right)(\xi-\lambda(1-\xi))}$,

with boundary conditions given by

$\left.S \frac{\mathrm{d} E}{\mathrm{~d} \xi}\right|_{\xi=0}-\frac{B x_{\mathrm{a} \infty} S}{h_{\infty}(0)^{2}} E(0)=\frac{B}{h_{\infty}(0)}\left(1-\frac{h_{\infty}(0) S}{B}\right)(1+\lambda)$,

In the following subsections we treat separately flows with and without a yield stress. Although there is common methodology between these two subsections, the details of the calculations are different in important ways that are linked to the differing curvatures at the fronts of the flows.

\subsection{Yield stress flows $(B>0)$}

We substitute (4.1) into (2.7) and linearise on the assumption that $h_{\infty}(\xi) \gg \epsilon \tilde{h}(\xi, t)$, which is certainly valid away from the front provided $\epsilon$ is sufficiently small. At the front $(\xi=1), h_{\infty}$ vanishes and it will be shown below that the solution in this region requires special attention. However sufficiently distant from the front, we obtain the following expression at leading order

$$
\begin{aligned}
\frac{\partial \tilde{h}}{\partial t}- & \frac{B}{h_{\infty}}\left(1-\frac{h_{\infty} S}{B}\right)\left(\xi \frac{\mathrm{d} \tilde{x}_{\mathrm{a}}}{\mathrm{d} t}-\frac{\mathrm{d} \tilde{x}_{\mathrm{y}}}{\mathrm{d} t}\right)=-\frac{n}{1+n} \frac{\epsilon^{1 / n} B^{1 / n}}{x_{\mathrm{a} \infty}} \frac{\partial}{\partial \xi} \\
\times & {\left[h_{\infty}^{2}\left(\frac{\tilde{h}}{h_{\infty}}+\left(1-\frac{h_{\infty} S}{B}\right) \frac{\tilde{x}_{\mathrm{a}}}{x_{\mathrm{a} \infty}}-\frac{h_{\infty}}{B x_{\mathrm{a} \infty}} \frac{\partial \tilde{h}}{\partial \xi}\right)^{1+1 / n}\right] . }
\end{aligned}
$$

Importantly we note that the leading order terms in the perturbation variables on the right-hand side of (4.3) have exponent $1+1 / n$, whereas those on the left-hand side are of exponent 1 . The leading order form of the boundary conditions are no flux of fluid at $\xi=0$, which corresponds to

$$
\left.\frac{\partial \tilde{h}}{\partial \xi}\right|_{\xi=0}-\frac{B x_{\mathrm{a} \infty}}{h_{\infty}(0)^{2}} \tilde{h}(0, t)=\frac{B}{h_{\infty}(0)}\left(1-\frac{h_{\infty}(0) S}{B}\right) \tilde{x}_{\mathrm{a}} .
$$

At the front of the flow we enforce $h(1, t)=0$ for all times and this forces the perturbation to the height profile to vanish here,

$\tilde{h}(1, t)=0$.

$E(1)=0$,

and if $B>B_{\mathrm{c}}$ and hence $\lambda \neq 0$,

$E(0)=\lambda$.

The left-hand side of (4.10) is solely a function of time and the right-hand side solely a function of $\xi$ so they must both be equal to a constant, $-\alpha \epsilon^{1 / n}$, and we obtain an ordinary differential equation for $F(t)$,

$\frac{1}{F^{1+1 / n}} \frac{\mathrm{d} F}{\mathrm{~d} t}=-\alpha \epsilon^{1 / n} \equiv-\frac{n}{1+n} \epsilon^{1 / n} S^{(2+n) / n} \frac{1}{k(B, S)}$,

where $k$ is a separation constant that is a function of $B$ and $S$. Integration of this equation leads us to the solution:

$F(t)=\frac{1}{\epsilon}\left(\frac{n}{\alpha t}\right)^{n}$,

where the constant of integration has been set to zero. This is appropriate because the constant sets the origin of time, but we are only interested in large time so we may choose it arbitrarily. We note that $\alpha$ must be positive to allow this solution to be be valid for arbitrary $n$. We also note that $F \propto \epsilon^{-1}$-and so the perturbation $\epsilon \tilde{h}$ becomes independent of $\epsilon$, as required, because $\epsilon$ is an arbitrary ordering parameter.

In order to complete the solution we must solve the spatial ordinary differential equation given by setting the right-hand side of (4.10) equal to $-\alpha \epsilon^{1 / n}$ and solving with appropriate boundary conditions in order to determine the value of $-\alpha$. This is subsequently done in the remainder of this section but we already have demonstrated an important result: all the perturbation variables decrease as $1 / t^{n}$ for $t \gg 1$. 
We may simplify the differential equation governing $E(\xi)$ and determine $k$ and $\lambda$ by making the following change of independent variable: we take the variable $E(\xi)$ to be a function of

$W \equiv W\left(-\mathrm{e}^{-1-S^{2} x_{\mathrm{a} \infty}(1-\xi) / B}\right)$,

where $W$ denotes the appropriate real-valued branch of the Lambert- $W$ function (i.e $W \equiv W_{0}$ when $S>0$ and $W \equiv W_{-1}$ when $S<0)$. The yield point, or back of the channel, at $\xi=0$ corresponds to $W=W_{\mathrm{y}} \equiv W\left(-\mathrm{e}^{-1-S^{2} x_{\mathrm{a} \infty} / B}\right)$, while the front of the current at $\xi=1$ corresponds to $W=-1$. Furthermore we substitute

$E(\xi)=\frac{W}{1+W}[P(W)-1+(1+\lambda)(1-\xi)]$.

Then the differential equation becomes

$P=k\left[(1+W)^{2}\left[-\frac{W^{2}}{1+W} P^{\prime}\right]^{1+1 / n}\right]^{\prime}$,

where a prime denotes differentiation with respect to $W$. The boundary condition of vanishing height at the front $(\xi=1)$ becomes $P(-1)=1$ : in fact this is the only possible condition that keeps $E(1)$, and thus $\tilde{h}(1, t)$, finite. The condition of no-flux at the rear of the active region become $P^{\prime}\left(W_{\mathrm{y}}\right)=0$. For $B<B_{\mathrm{c}}$ these are sufficient to determine the solution since the active region includes all of the fluid. However if $B>B_{\mathrm{c}}$, we complete the solution by the condition $\lambda=W_{\mathrm{y}} P\left(W_{\mathrm{y}}\right)$. lem,

Finally we make one further substitution to simplify the prob-

$I(W)=\int_{-1}^{W} P(W) \mathrm{d} W$

which yields

$\frac{W^{2}}{1+W} I^{\prime \prime}+K\left[\frac{I}{(1+W)^{2}}\right]^{n /(n+1)}=0$,

where $K=k^{-n /(n+1)}$, subject to $I(-1)=0, I^{\prime}(-1)=1$ and $I\left(W_{\mathrm{y}}\right)=0$. (Additionally if $B>B_{\mathrm{c}}, \lambda=W_{\mathrm{y}} I^{\prime}\left(W_{\mathrm{y}}\right)$.) Thus the problem is reduced to a relatively straightforward two point eigenvalue problem, which can be readily integrated numerically to determined the separation constant, $k$ and the shape of the perturbation to the profile, $\tilde{h}$.

In Table 1 we list the values of the separation constant, $K \equiv$ $k^{-n /(1+n)}$ and $\lambda$ for various values of the flow index, $n$ when $S=1$ and (a) $B=0.5$ and (b) $B=2$. The corresponding functions $I(W)$ are plotted in Fig. 8. We also plot the shape of the perturbation to the profile of the current (Fig. 9) for $n=1 / 3,1$ and 3, noting that the perturbations exhibit highest curvature towards the front of the current. As a consequence it will be shown that the perturbation to the height field, $\epsilon \tilde{h}$, is actually larger than the final arrested state, $h_{\infty}$, in the region close to the front, which implies that the linearisation underlying these results fails and the analysis requires more careful consideration.

It is straightforward to solve the eigenvalue differential equation in the limits $n \rightarrow 0$ and $n \rightarrow \infty$. First, when $n \gg 1$, the governing equation becomes linear to leading order and is given by

$I^{\prime \prime}+\frac{K}{W^{2}(1+W)} I=0$.

This may be integrated straightforwardly and the solution may be expressed in terms of hypergeometric functions; the eigenvalue $K$ and $\lambda$ are given in Table 1 , for $S=1$ and $B=0.5,2$.

When $n \ll 1$, the governing equation may be analysed using matched asymptotic expansion where the 'outer' region $-1 \leq$ $W \ll W_{\mathrm{y}}$ is matched to an 'inner' region close to the end of the
Table 1

The separation constant $K \equiv k^{-n /(n+1)}$ and $\lambda$ at various values of $n$ for $S=1$ and (a) $B=0.5$; (b) $B=2$

\begin{tabular}{|c|c|c|}
\hline$n$ & $K$ & \\
\hline \multicolumn{3}{|l|}{ (a) } \\
\hline 0 & 0.1816 & \\
\hline $1 / 10$ & 0.1904 & \\
\hline $1 / 5$ & 0.1986 & \\
\hline $1 / 3$ & 0.2085 & \\
\hline $1 / 2$ & 0.2195 & \\
\hline 1 & 0.2459 & \\
\hline 2 & 0.2802 & \\
\hline 3 & 0.3016 & \\
\hline 5 & 0.3268 & \\
\hline 10 & 0.3538 & \\
\hline$\infty$ & 0.3931 & \\
\hline$n$ & $K$ & $\lambda$ \\
\hline \multicolumn{3}{|l|}{ (b) } \\
\hline 0 & 4.2776 & 1.1388 \\
\hline $1 / 10$ & 4.0910 & 1.0173 \\
\hline $1 / 5$ & 3.9403 & 0.9254 \\
\hline $1 / 3$ & 3.7794 & 0.8333 \\
\hline $1 / 2$ & 3.6237 & 0.7490 \\
\hline 1 & 3.3278 & 0.6001 \\
\hline 2 & 3.0542 & 0.4716 \\
\hline 3 & 2.9265 & 0.4135 \\
\hline 5 & 2.8059 & 0.3592 \\
\hline 10 & 2.7033 & 0.3126 \\
\hline$\infty$ & 2.5906 & 0.2601 \\
\hline
\end{tabular}
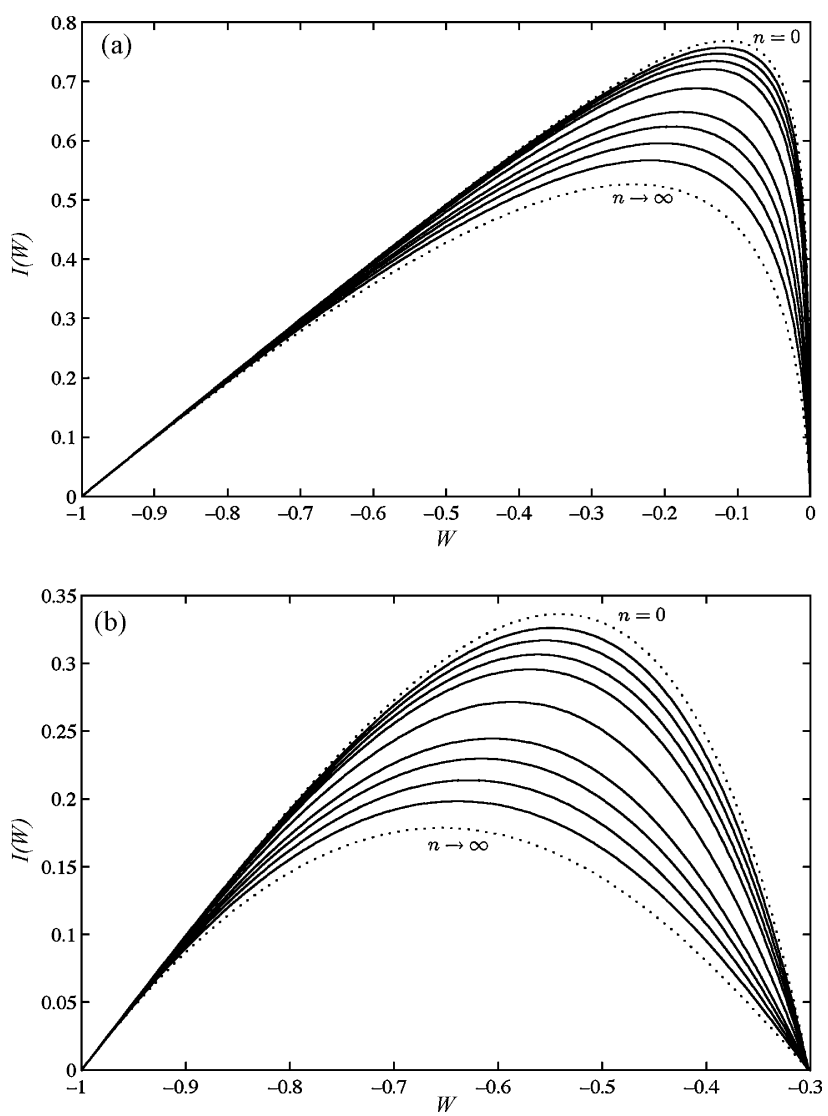

Fig. 8. The perturbation $I(W)$ as a function of $W$ for $S=1$ and (a) $B=0.5$; (b) $B=2$. The function $I(W)$ is determined for $n=10,5,3,2,1,1 / 2,1 / 3,1 / 5,1 / 10(-)$. Also plotted are the asymptotic profiles when $n=0$ and $n \rightarrow \infty(\cdots)$. 

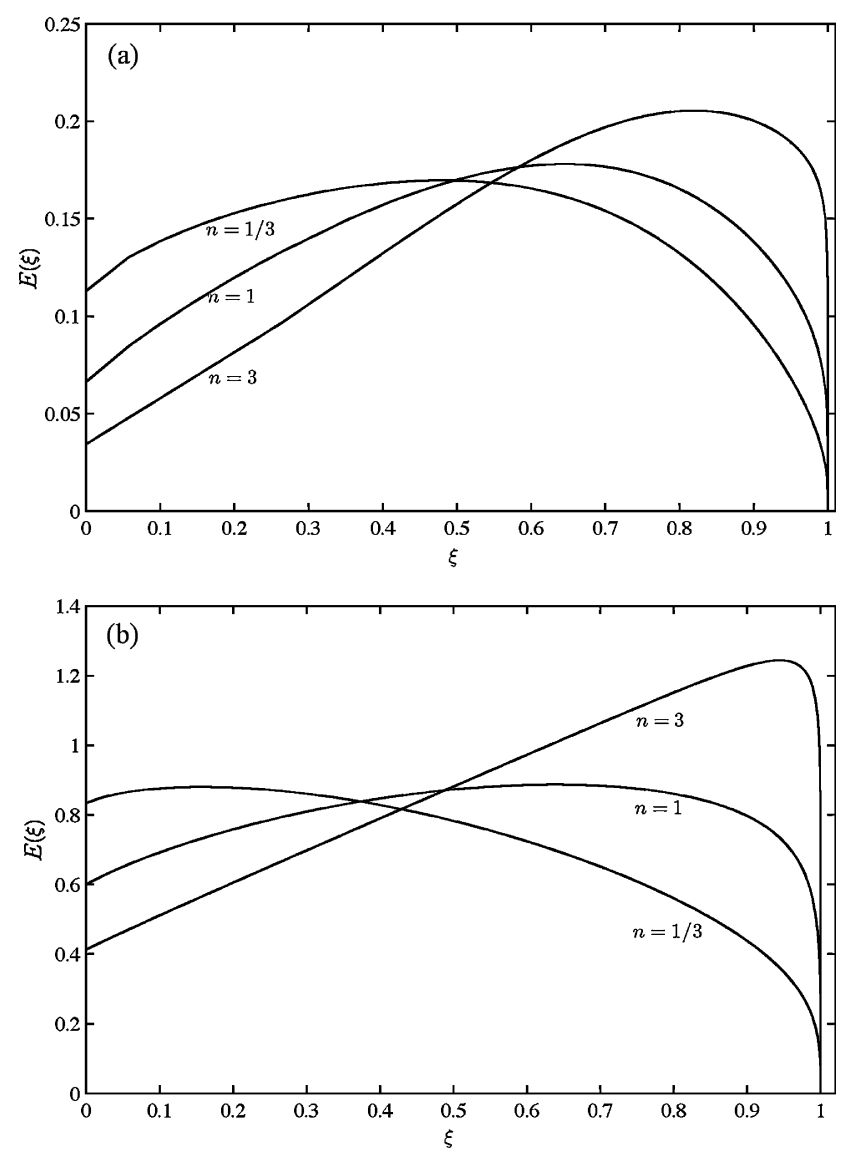

Fig. 9. The perturbation $E(\xi)$ as a function of the rescaled distance $\xi$ for $S=1$ and (a) $B=0.5$; (b) $B=2$. The function $I(W)$ is determined for $n=3,1,1 / 3$. $h_{\infty}=\left(2 B x_{\mathrm{a} \infty}(1-\xi)\right)^{1 / 2}+\cdots \quad$ and

$\tilde{h}=S F(t) C\left(\frac{2 S^{2}}{B} x_{\mathrm{a} \infty}(1-\xi)\right)^{1 /[2(n+1)]}$,

where $C=(n+1) k^{-n /(n+1)} /(n+2)$. Thus $h_{\infty} \sim \epsilon \tilde{h}$ when

$2 x_{\mathrm{a} \infty}(1-\xi) \sim(\epsilon C F)^{2(n+1) / n}\left(\frac{S^{2}}{B}\right)^{(n+2) / n}$.

This identifies a region close to the front of the slumping fluid within which the leading order height, $h_{\infty}(\xi)$, and the perturbation, $\epsilon \tilde{h}(\xi, t)$, are of the same order of magnitude. This means that the linearizarion used to derive (4.10) is no longer valid; instead the equations must be analysed within a new asymptotic region close to the front and matched to the solutions in the interior of the fluid. To this end, we define a rescaled coordinate,

$\eta=\frac{2 x_{\mathrm{a} \infty}(1-\xi)}{(\epsilon C F)^{2(n+1) / n}\left(S^{2} / B\right)^{(n+2) / n}}$.

We observe because $F \rightarrow 0$ as $t \rightarrow \infty$, this rescaled spatial coordinate identifies a 'boundary layer' close to the front $(\xi=1)$, which is of progressively diminishing magnitude. The final arrested state and the perturbation are comparable when $\eta=O(1)$, both being $O\left(\epsilon^{1+1 / n}\right)$. Explicitly, we can show that

$h_{\infty} \sim(\epsilon F C)^{1+1 / n} S^{1+2 / n} B^{-1 / n} \eta^{1 / 2}$,

$\epsilon \tilde{h} \sim(\epsilon F C)^{1+1 / n} S^{1+2 / n} B^{-1 / n} \eta^{1 /[2(n+1)]}$.

We now analyse the governing Eq. (2.7) in terms of independent variables $\eta$ and $t$ : we substitute the leading order expressions for $h_{\infty}$ and we introduce

$\epsilon \tilde{h}=(\epsilon F C)^{1+1 / n} S^{1+2 / n} B^{-1 / n} H(\eta)$.

This form is consistent with the magnitude of the separable solution in the interior of the flow and within the 'boundary layer', we find the leading order equation is given by

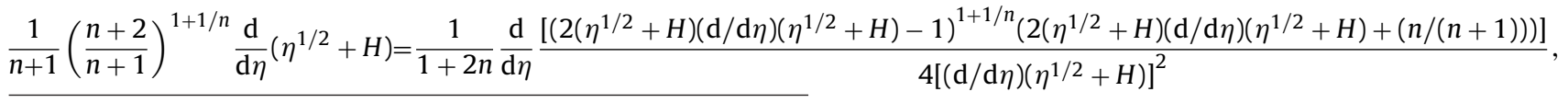

domain at $W=W_{\mathrm{y}}$. Within the outer region, to leading order we find that

$I=K(1-W) \log (-W)+(2 K+1)(W+1)$

Matching to the inner region demands that this expression vanishes at $W=W_{\mathrm{y}}$ and thus

$$
\begin{aligned}
& K=\frac{-\left(1+W_{\mathrm{y}}\right)}{\left(1-W_{\mathrm{y}}\right) \log \left(-W_{\mathrm{y}}\right)+2\left(1+W_{\mathrm{y}}\right)} \text { and } \\
& \lambda=\frac{2 W_{\mathrm{y}} \log \left(-W_{\mathrm{y}}\right)-1+W_{\mathrm{y}}^{2}}{\left(1-W_{\mathrm{y}}\right) \log \left(-W_{\mathrm{y}}\right)+2\left(1+W_{\mathrm{y}}\right)} .
\end{aligned}
$$

These asymptotic values agree closely with numerically determined ones in the regime $n \ll 1$.

The separable solution has determined the form of the perturbation to the height and length of the flow as the arrested state is approached under the assumption that $h_{\infty} \gg \epsilon \tilde{h}$. While this regime holds in the bulk of the current, it becomes invalid as the front is approached. This can be demonstrated by examining the behaviour of $E$ in the regime $1-\xi \ll 1$, or equivalent $I$ in the regime $1+W \ll 1$. We find that subject to the boundary condition that the height vanishes at the front, $H(0)=0$ and the matching condition that $H \rightarrow \eta^{1 /[2(n+1)]}$ as $\eta \rightarrow \infty$. It may be readily verified that this matching condition is automatically satisfied as the far-field asymptotic of this governing Eq. (4.30), thus confirming the approach above that allows the separation constant to be determined without explicitly calculating the profile within this boundary layer close to the front. The point $\eta=0$ is a singular point of the differential equation and we may determine analytically the behaviour in the region $\eta \ll 1$,

$H(\eta)=\frac{(n+2)(2 n+1)^{n /(n+2)}}{2^{1 /(n+2)}(n+1)^{(n+1) /(n+2)}} \eta^{1 /(n+2)}+\cdots$

Thus the ordinary differential equation may be integrated numerically from $\eta=\delta \ll 1$ to determine fully the perturbation to height field, $\tilde{h}$ within the region close to the front. We plot the perturbation, $H(\eta)$ in Fig. 10 for $n=1 / 3,1$ and 3.

It is noteworthy that when $\eta \ll 1, H \propto \eta^{1 /(n+2)}$ whereas $h_{\infty} \propto$ $\eta^{1 / 2}$. Thus as anticipated, sufficiently close to the front the perturbation dominates the arrested state-and therefore this asymptotic analysis is required to determine fully the approach to the arrested state. This behaviour close to the front, however, can be anticipated. An intruding free-surface flow of a fluid with power law rheology exhibits $h \sim\left(x_{\mathrm{f}}-x\right)^{1 /(n+2)}$ (see, for example [17,26,27]), whereas the arrested state has $h \sim\left(x_{\mathrm{f}}-x\right)^{1 / 2}$. Thus as the flow of a 


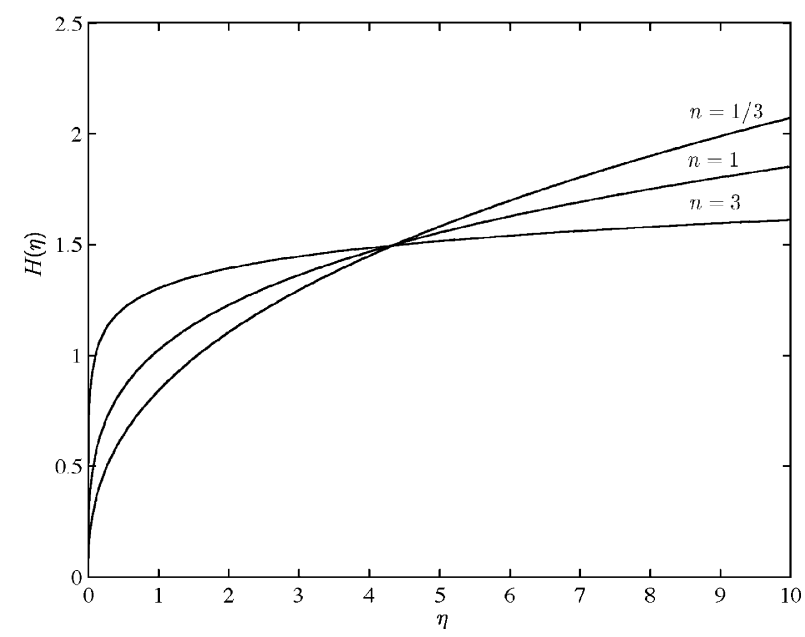

Fig. 10. The perturbation function, $H$, within the 'boundary layer' close to the front of the flow, as a function of the rescaled spatial coordinate, $\eta$, for flow indices $n=1 / 3$, 1 and 3.

Herschel-Bulkley fluid approaches its arrested state, although the interior becomes close to $h_{\infty}$, there exists a diminishing region close to the front within which $h_{\infty}$ does not provide the dominant shape of the interface. Rather, the material here is still flowing and its viscosity exhibits a dependence on the rate of strain similar to a power law fluid: this requires that $h \sim\left(x_{\mathrm{f}}-x\right)^{1 /(n+2)}$. This transition is precisely encompassed by the matched asymptotic analysis developed above.

\subsection{Slumps of fluids in the absence of a yield stress $(B=0)$}

Slumps of viscous fluid, released from rest behind a dam and flowing up an inclined plane, reach an arrested final state in the absence of a yield stress with the free surface lying horizontally. In this subsection we analyse the approach of such motion towards to the end state. Much of the methodology is similar to Section 4.1, but the detailed calculations work out somewhat differently. In fact it is clear from the results of Section 3 that a different approach will be required when $B=0$, because this is a singular limit of the governing equations. In physical terms, the difference arises because in the absence of a yield stress the final state is a horizontal surface, whereas with a yield stress $(B>0)$ the arrested state exhibits considerable curvature, especially close to the front.

For flows with vanishing yield stress $(B=0)$, the governing equation for the evolution of the shape of the free surface, $h(\xi, t)$, may be deduced from (2.7) and is given by

$\frac{\partial h}{\partial t}-\frac{\dot{x}_{\mathrm{f}}}{x_{\mathrm{f}}} \xi \frac{\partial h}{\partial \xi}=-\frac{n}{1+2 n} \frac{1}{x_{\mathrm{f}}} \frac{\partial}{\partial \xi}\left[h^{2+1 / n}\left(-\Lambda-\frac{1}{x_{\mathrm{f}}} \frac{\partial h}{\partial \xi}\right)^{1 / n}\right]$,

where we have substituted $-S=\Lambda>0$. The final state and the length of the intrusion along the inclined plane are given by (3.15). The evolution equation is subject to the following boundary conditions: no flow at the back wall of the lock, $\partial h / \partial x=-\Lambda$, at $\xi=0$; vanishing height at the front, $h=0$ at $\xi=1$; and conservation of mass per unit width

$x_{\mathrm{f}} \int_{0}^{1} h \mathrm{~d} \xi=\frac{1}{2}(2-\Lambda)$.

We analyse how this arrested state is approached by introducing

$h=h_{\infty}+\epsilon \tilde{h} \quad$ and $\quad x_{\mathrm{f}}=x_{\mathrm{f} \infty}-\epsilon \tilde{x}_{\mathrm{f}}$, where $\epsilon$ is an ordering parameter. On the assumption that $\epsilon \ll 1$ and that $h_{\infty} \gg \epsilon \tilde{h}$, we obtain the following linearised equation:

$$
\begin{aligned}
\epsilon \frac{\partial \tilde{h}}{\partial t}-\epsilon \frac{\dot{\tilde{x}}_{\mathrm{f}}}{x_{\mathrm{f} \infty}} \frac{\partial h_{\infty}}{\partial \xi}= & -\epsilon^{1 / n} \frac{n}{1+2 n} \Lambda^{2+1 / n} x_{\mathrm{f} \infty} \frac{\partial}{\partial \xi} \\
& \times\left[(1-\xi)^{2+1 / n}\left(-\frac{\partial \tilde{h}}{\partial \xi}+\tilde{x}_{\mathrm{f}} \Lambda\right)^{1 / n}\right],
\end{aligned}
$$

subject to $\partial \tilde{h} / \partial \xi(0, t)=\Lambda \tilde{x}_{\mathrm{f}}, \tilde{h}(1, t)=0$ and

$\int_{0}^{1} \tilde{h} \mathrm{~d} \xi=\frac{1}{2} \Lambda \tilde{x}_{\mathrm{f}}$

We seek a solution of the form $\tilde{h}=\Lambda \tilde{x}_{\mathrm{f}}+g_{1}(t) g_{2}(\xi)$, but it is not possible to find a separable solution, $g_{2}(\xi)$, that remains finite at both $\xi=0$ and $\xi=1$. However, while the solution $\tilde{h}=\Lambda \tilde{x}_{\mathrm{f}}$ satisfies the conditions for no flow at the back wall and conservation of mass per unit volume, it does not give a vanishing height at the front and thus can not represent the complete solution. Furthermore in contrast to the analysis of Section 4.1, the linearised governing equation does not permit $\tilde{x}_{\mathrm{f}}(t)$ to be calculated.

The resolution of this difficulty is that the governing equation can not be linearised close to the front $(\xi=1)$, because in that region we find that $h_{\infty} \sim \epsilon \tilde{h}$ (cf. Section 4.1). Thus the solution $\tilde{h}=\Lambda \tilde{x}_{\mathrm{f}}$ is appropriate sufficiently distant from the front, but a different approach is required within the region close to the front. The size of this region may be estimated as follows:

$\Lambda x_{\mathrm{f} \infty}(1-\xi) \sim \epsilon \Lambda \tilde{x}_{\mathrm{f}}$.

Thus as $\tilde{x}_{\mathrm{f}}$ is a decreasing function of time, there is a progressively diminishing boundary layer close to the front within which the arrested final profile and perturbation are of the same order of magnitude. We analyse this boundary layer by introducing a rescaled spatial coordinate

$\eta=\frac{(1-\xi)}{\epsilon X_{\mathrm{f} \infty} \tilde{x}_{\mathrm{f}}}$.

Within the boundary layer, $h_{\infty}=\epsilon \Lambda \tilde{x}_{\mathrm{f}} \eta$ and at the edge of the region the perturbation must match with the solution in the interior. Adopting $\eta$ and $t$ as independent variables and substituting $\tilde{h}=\epsilon \Lambda \tilde{x}_{\mathrm{f}}(t) \hat{H}(\eta)$, the leading order terms of the governing equation are given by

$$
\begin{aligned}
\dot{\tilde{x}}_{\mathrm{f}}\left[\hat{H}-(\eta+1) \frac{\mathrm{d} \hat{H}}{\mathrm{~d} \eta}-1\right]= & \frac{n}{1+2 n} \epsilon^{1 / n} \Lambda^{1+2 / n} \tilde{x}_{\mathrm{f}}^{1+1 / n} \frac{\mathrm{d}}{\mathrm{d} \eta} \\
& \times\left[(\eta+\hat{H})^{2+1 / n}\left(\frac{\mathrm{d} \hat{H}}{\mathrm{~d} \eta}\right)^{1 / n}\right],
\end{aligned}
$$

subject to $\hat{H}(0)=0$ and $\hat{H} \rightarrow 1$ as $\eta \rightarrow \infty$. We may now separate the functions of the independent variables and thus

$\frac{\dot{\tilde{x}}_{\mathrm{f}}}{\tilde{x}_{\mathrm{f}}^{1+1 / n}}=-\frac{n}{1+2 n} \epsilon^{1 / n} \Lambda^{1+2 / n} k$,

where $k$ is a separation constant depending on $n$. This yields

$\tilde{x}_{\mathrm{f}}=\frac{1}{\epsilon}\left(\frac{1+2 n}{\Lambda^{1+2 / n} k t}\right)^{n}$.

Hence the dependence of the perturbation upon $t^{-n}$ is identical to flows with a yield stress (see Section 4.1). Also we note that the perturbation $\epsilon \tilde{h}$ is independent of $\epsilon$, as is to be expected because $\epsilon$ 


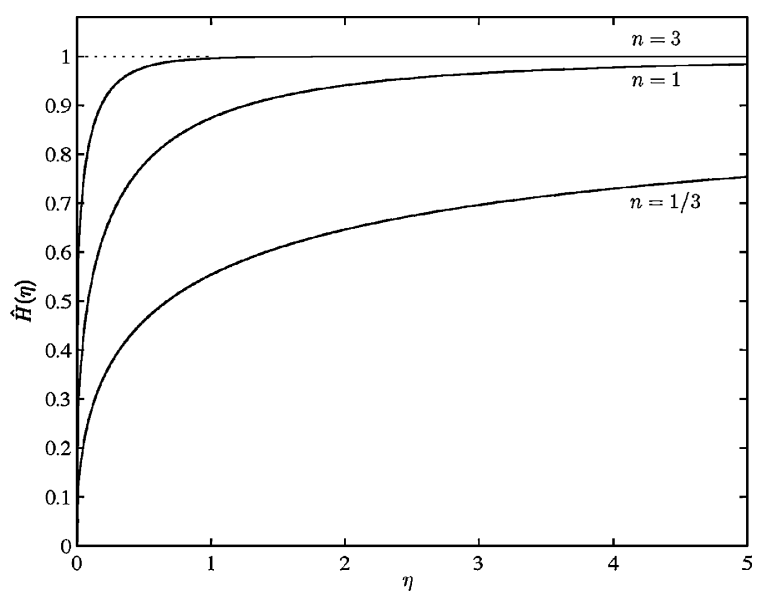

Fig. 11. The perturbation function, $\hat{H}$, within the 'boundary layer' close to the front of the flow, as a function of the rescaled spatial coordinate, $\eta$, for flow indices $n=1 / 3,1$ and 3 in the absence of a yield stress $(B=0)$.

\section{Table 2}

The separation constant $k$ at various values of $n$ in the absence of a yield stress $(B=0)$

\begin{tabular}{ll}
\hline$n$ & $k$ \\
\hline $1 / 5$ & 0.0001993 \\
$1 / 3$ & 0.01840 \\
1 & 0.6090 \\
3 & 1.037 \\
5 & 1.062 \\
\hline
\end{tabular}

is an arbitary ordering parameter. The spatial dependence reduces to

$$
-k\left[\hat{H}-(\eta+1) \frac{\mathrm{d} \hat{H}}{\mathrm{~d} \eta}-1\right]=\frac{\mathrm{d}}{\mathrm{d} \eta}\left[(\eta+\hat{H})^{2+1 / n}\left(\frac{\mathrm{d} \hat{H}}{\mathrm{~d} \eta}\right)^{1 / n}\right] .
$$

In the regime $\eta \ll 1, \hat{H}(\eta)=k^{n /(n+2)}(n+2)^{1 /(n+2)} \eta^{1 /(n+2)}+\cdots$ and so the complete solution may be computed by numerically shooting from $\eta=\delta \ll 1$ to the far-field and adjusting to the value of $k$ until the matching condition is satisfied. We plot the form of the perturbation field, $\hat{H}(\eta)$ in Fig. 11 for $n=1 / 3,1$ and 3 and give values for the separation constant $k$ in Table 2 .

Once again, we note that the spatial dependence close to front: when the current is flowing, we require $h \sim\left(x_{\mathrm{f}}-x\right)^{1 /(n+2)}$ (see above and [17]) within a diminishing boundary layer as the current slows and approaches its final state in which $h_{\infty} \sim\left(x_{\mathrm{f}}-x\right)$.

\section{Summary and conclusions}

In this contribution we have analysed the motion and arrest of fluid released from rest behind a dam and allowed to flow along an inclined, two-dimensional channel. We have fully characterised the final arrested profile of the free surface in terms of two dimensionless parameters, $S$ and $B$, that measure the magnitude of the inclination of the channel relative to the initial aspect ratio of the release and the magnitude of the yield stress of the material relative to the weight of the fluid layer, respectively. This final profile can be expressed in a compact form in terms of Lambert- $W$ functions, with the two real-valued branches representing slumps up and downhill. This representation aids the subsequent analysis in this paper as the approach to the arrested state is considered. Following release, it is possible that not all of the fluid is set in motion before the stresses drop below the yield stress and the flow arrests. We show that from dam-break initial conditions we may evaluate a critical value of the yield stress as a function of the slope, expressed as $B_{\mathrm{c}}(S)$ in dimensionless variables, such that there is an interior yield point if this parameter is exceeded.

Turning then to the approach towards the final state, we have generalised the results for flows along horizontal channels [17] to show that the state is only attained asymptotically as $t \rightarrow \infty$ and that perturbations decay as $t^{-n}$. Furthermore we calculate the constant of proportionality (the 'separation' constant) in this decay by solving numerically a relatively simple eigenvalue problem. These analyses and results do indicate an important feature that was not noticed before, namely that the perturbation to the final profile is larger than the final profile itself as the front is approached. This can be anticipated because in the region $\left(x_{\mathrm{f}_{\infty}}-x\right) / x_{\mathrm{f}_{\infty}} \ll 1, h_{\infty} \sim$ $\left(x_{\mathrm{f} \infty}-x\right)^{1 / 2}$ while the flowing state must exhibit $h \sim\left(x_{\mathrm{f}}-x\right)^{1 /(n+2)}$. The resolution of this difficulty is to introduce a new asymptotic region, within a diminishing boundary layer close to the front, and to match to the interior. It was shown that its effect on the solution and the calculation of the decay is sub-dominant unless $S<0$ and $B=0$, in which case the motion upslope towards a stationary state with a horizontal interface is crucially dependent upon the profile close to the front.

The algebraic decay as the arrested state is approached has been seen in numerical computations $[7,16]$ and there is some evidence of it in recent experiments [21]. It has some important consequences in the interpretation of data from devices such as the Bostwick consistometer or other slump-like test, in which material is set into motion and allowed to flow towards a yield-stress arrested state. The algebraic decay means that unlike the cessation of flows of yield stress materials in pipes and channels, for which when the driving pressure gradient is abruptly removed and the flow stops in a finite time [28], the free-surface slumps only approach the arrested state asymptotically.

There are dynamical features that have not been included in this model and which warrant investigation. For instance, the inertia of the flowing material may become non-negligible at some stage during the motion; surface tension effects could become significant in regions of significant curvature; and these predictions could be modified during the initial phases of the motion when the flow is not shallow and the pressure not hydrostatic. Alternatively, and this may be required to explain the experimental observations of concentrated suspensions of particles and clay dispersions that appear to arrest abruptly (e.g. [22]), it would be interesting to analyse the flow and arrest of systems under modified constitutive laws that account in some way for the micromechanics of the particulate materials.

\section{Appendix A. Properties of the Lambert- $W$ function}

The Lambert- $W$ function is defined to be the function satisfying

$W(z) \exp (W(z))=z$

(see [25]). The function has a branch point at $z=-\mathrm{e}^{-1}$ and two principal, real-valued branches: $W_{0}(z)$ defined for $z>-\mathrm{e}^{-1}$ and $W_{-1}(z)$ defined for $0<z<-\mathrm{e}^{-1}$ (see Fig. 12).

From (A.1), it is straightforward to show that

$\frac{\mathrm{d} W}{\mathrm{~d} z}=\frac{W}{(W+1) z}$.

Furthermore, when e $z+1 \ll 1$,

$W(z)=-1+(2(\mathrm{e} z+1))^{1 / 2}-\frac{2}{3}(\mathrm{e} z+1)+\cdots$,

and when $|z| \ll 1$,

$W_{0} \sim z$ and $W_{-1} \sim \log z$. 


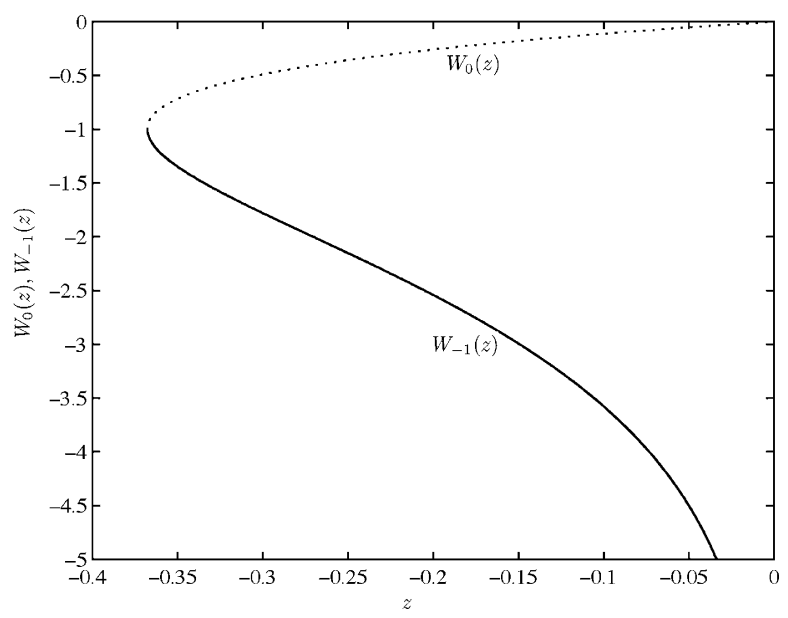

Fig. 12. The real-valued branches of the Lambert- $W$ function, $W_{0}(z)$ and $W_{-1}(z)$, in the range $z<0$.

\section{References}

[1] C. Ancey, Plasticity and geophysical flows: a review, J. Non-Newtonian Fluid Mech. 142 (2007) 4-35.

[2] R.W. Griffiths, The dynamics of lava flow, Ann. Rev. Fluid Mech. 32 (2000) 477-518.

[3] N.J. Balmforth, R.V. Craster, Geophysical aspects of non-Newtonian fluid mechanics, in: N.J. Balmforth, A. Provenzale (Eds.), Geomorphological Fluid Mechanics (Lecture Notes in Physics), Springer, 2002, p. 589.

[4] N.J. Balmforth, R.V. Craster, A consistent thin-layer theory for Bingham plastics, J. Non-Newtonian Fluid Mech. 84 (1999) 65-81.

[5] P. Perona, Bostwick degree and rheological properties: an up-to-date viewpoint, Appl. Rheol. 15 (2005) 218-229.

[6] R.R. Milczarek, K.L. McCarthy, Relationship between the Bostwick measurement and fluid properties, J. Texture Stud. 37 (2006) 640-654.

[7] N.J. Balmforth, R.V.Craster, P. Perona, A.C. Rust, R. Sassi, Viscoplastic dam breaks and the Bostwick consistometer, J. Non-Newtonian Fluid Mech. 142 (2007) 63-78.
[8] A. Ritter, Die Fortpflanzung der Wasserwellen, Zeitschrift des Vereines Deutscher Ingenieure 36 (33) (1892) 947-954.

[9] B. Hunt, An inviscid dam-break solution, J. Hydraul. Res. 25 (1987) 313-327.

[10] A.J. Hogg, D. Pritchard, The effects of drag on dam-break and other shallow inertial flows, J. Fluid Mech. 501 (2004) 179-212.

[11] H.E. Huppert, The propagation of two-dimensional and axisymmetric viscous gravity currents over a rigid horizontal surface, J. Fluid Mech. 121 (1982) 43-58.

[12] H.E. Huppert, Flow and instability of a viscous current down a slope, Nature 300 (1982) 427-499.

[13] G. Lube, H.E. Huppert, R.S.J. Sparks, A. Freundt, Collapses of two-dimensional granular columns, Phys. Rev. E 72 (2005) 041301.

[14] N.J. Balmforth, R.R. Kerswell, Granular collapse in two dimensions, J. Fluid Mech. 538 (2005) 399-428.

[15] A.J. Hogg, Two-dimensional granular slumps down slopes, Phys. Fluids (2007) 19.

[16] X. Huang, M.H. Garcia, A Herschel-Bulkley model for mud flow down a slope, J. Fluid Mech. 364 (1998) 305-333.

[17] G.P. Matson, A.J. Hogg, Two-dimensional dam break flows of Herschel-Bulkley fluids: the approach to the arrested state, J. Non-Newtonian Fluid Mech. 142 (2007) 79-94.

[18] K.F. Liu, C.C. Mei, Slow spreading of a sheet of Bingham fluid on an inclined plane, J. Fluid Mech. 207 (1989) 505-529.

[19] N.J. Balmforth, R.V. Craster, A.C. Rust, R. Sassi, Viscoplastic flow over an inclined surface, J. Non-Newtonian Fluid Mech. 142 (2007) 219-243.

[20] H. Chanson, S. Jarny, P. Coussot, Dam break wave of thixotropic fluid, J. Hydraul. Eng. 132 (2006) 280-293.

[21] S. Cochard, Measurements of time-dependent free-surface viscoplastic flows down steep slopes, PhD thesis, Ecole Polytechnique Fédérale de Lausanne, 2007.

[22] M.A. Hallworth, H.E. Huppert, Abrupt transitions in high-concentration, particle-driven gravity currents, Phys. Fluids 10 (1998) 1083-1087.

[23] C. Ancey, H. Jorrot, Yield stress for particle suspensions within a clay dispersion, J. Rheol. 45 (2001) 297-319.

[24] L.M. Hocking, The spreading of a thin drop by gravity and capillarity, Q. J. Mech Appl. Math. 36 (1983) 55-69.

[25] R.M. Corless, G.H. Gonnet, D.E.G. Hare, D.J. Jeffrey, D.E. Knuth, On the Lambert Wfunction, Adv. Comput. Math. 5 (1996) 329-359.

[26] J. Gratton, F. Minotti, S.M. Mahajan, Theory of creeping gravity currents of a non-Newtonian liquid, Phys. Rev. E 60 (1999) 6960-6967.

[27] C.A. Perazzo, J. Gratton, Thin film flow of non-Newtonian fluid on an incline, Phys. Rev. E 67 (2003) 016307.

[28] R.R. Huilgol, B. Mena, J.M. Piau, Finite stopping time problems and rheometry of Bingham fluids, J. Non-Newtonian Fluid Mech. 102 (2002) 97-107. 